\title{
Spatial short-term memory assists in maintaining occluded objects
}

\author{
HYUNKYU LEE \\ University of Illinois at Urbana-Champaign, Urbana, Illinois \\ AND \\ SHaUn P. Vecera \\ University of Iowa, Iowa City, Iowa
}

\begin{abstract}
We examined the role of spatial short-term memory (SSTM) in maintaining the hidden portions of occluded objects. We measured the degree of maintenance by asking participants to perform an object-based attention task on occluded objects while maintaining four locations in SSTM. SSTM did not interfere with object-based selection generally. Maintenance of occluded portions of objects was prevented when four locations were held in SSTM and these locations did not overlap with the locations of occluded objects. Importantly, when the observers remembered locations that overlapped with the occluded regions of objects, the occluded objects were maintained behind the occluder. These results suggest that SSTM participates in maintaining the occluded sections of objects.
\end{abstract}

Due to the movement of our body and objects in our environment, our visual system is confronted with visual information that contains objects that are fully visible at one moment and partially or totally occluded by other objects at another moment. However, both primate and nonprimate visual systems are highly efficient in representing and recognizing objects across the variability created by occlusion (for completion in nonhumans, see Fujita, 2001; Kanizsa, Renzi, Conte, Compostela, \& Guerani, 1993; Nagasaka \& Wasserman, 2008). Visual completion serves as a mediating mechanism, enabling objects to be completed behind occluding surfaces and allowing the occluded object to appear as a single object that continues behind the occluder (Kanizsa, 1975; Michotte, Thinès, \& Crabbé, 1964/1991).

Visual completion processes have been shown to operate quickly on the basis of bottom-up image information; in most situations, higher level cognitive operations are not required to infer the shape of an occluded object (e.g., Davis \& Driver, 1998; He \& Nakayama, 1992; Moore, Grosjean, \& Lleras, 2003; Rensink \& Enns, 1998). For example, in Figure 1A, observers subjectively experience an elongated occluded horse, although the percept violates the knowledge that such an animal is not possible in the real world (see Kanizsa \& Gerbino, 1982, for a similar demonstration). Image cues that support visual completion include good contour continuation (Wouterlood \& Boselie, 1992) and cotermination and T-junctions (Rensink \& Enns, 1998). Visual completion also has been described as the result of interpolating between the vis- ible segments of occluded objects (Kellman \& Shipley, 1991).

More objective results for potent image-based completion come from Pratt and Sekuler (2001), who demonstrated that previous exposure to a scene did not affect completion. In their experiments, observers saw a preview display containing four rectangles, followed by an occluder (Figure 1B). When the occluder was present, only the ends of the four rectangles were visible, and phenomenologically, the display appeared to contain two longer, occluded rectangles (not four separated rectangles). Next, a peripheral cue summoned attention to the end of one of the rectangles, followed by several shapes that appeared inside the rectangles. Observers were asked to report the identity of the largest shape. Such object-based attention tasks reveal faster responses to uncued (or invalidly cued) targets appearing at the other end of the cued object than to those invalidly cued targets appearing on the other, uncued object (Egly, Driver, \& Rafal, 1994; Vecera, 1994). Pratt and Sekuler showed that an object-based attentional benefit based on a completed image was present even when observers saw the preview display containing four distinct objects. These findings indicate that image cues such as T-junctions and colinear lines easily override observers' knowledge of four disconnected objects behind an occluder.

Although most research on visual completion supports a bottom-up view, some findings suggest that later visual processes can influence completion or the outputs of completion processes. Visual memory may comple-

S. P. Vecera, shaun-vecera@uiowa.edu 


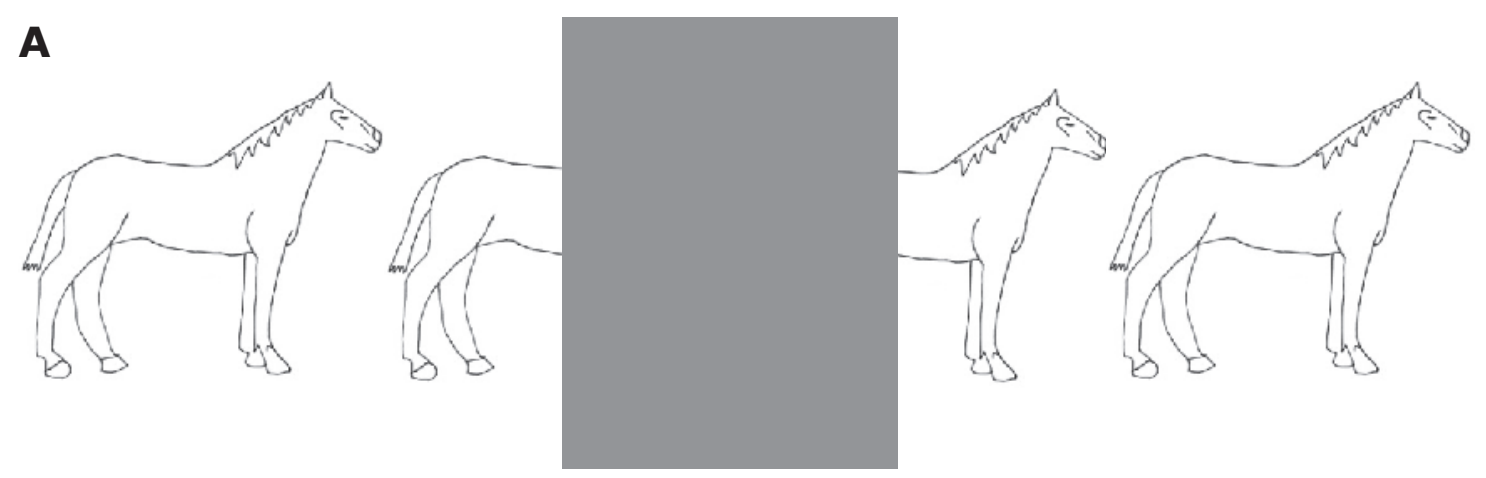

B

Pratt \& Sekuler (2001)

Lee \& Vecera (2005)
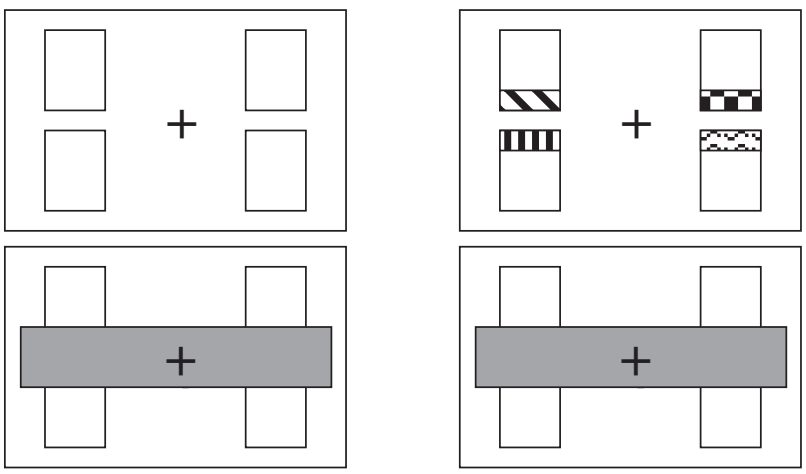

Figure 1. (A) An example supporting the bottom-up account of visual completion. (B) Displays from Pratt and Sekuler (2001) and Lee and Vecera (2005). Each pattern at the ends of the rectangles indicates a different color.

ment image information in completing occluded objects (e.g., Joseph \& Nakayama, 1999; Lee \& Vecera, 2005). We previously demonstrated that objects stored in visual short-term memory (VSTM) interfered with visual completion based on salient image features (Lee \& Vecera, 2005). Observers were presented with four unconnected rectangles, each of which had a color patch at its end (Figure 1B). An occluder appeared and covered the gaps that separated the rectangles, allowing the four rectangles to be completed into two longer rectangles. However, when observers stored the four color patches and rectangles in VSTM, there was no evidence of visual completion, even though the occluded display provided strong image cues for completion. These results suggest that higher level cognitive processes such as VSTM can influence visual completion. Actively remembering objects that were visible prior to occlusion can influence completion, allowing prior knowledge of the four rectangles to trump imagebased completion cues. VSTM could affect completion either by altering completion processes themselves or by allowing the memory representation to take priority over the perceptual representation based on completion processes.

In the present experiments, we investigated the maintenance of objects that were initially perceived and later occluded. When a previously visible object becomes occluded, perceptual cues alone (e.g., T-junctions) can sup- port the completion of the occluded portion of the object, as is widely discussed in the literature. But we highlight another possibility beyond completion alone: In addition to a completion process, when an object is fully visible and later occluded, the occluded portion of the object might be maintained in visual memory. (Such maintenance would certainly be necessary for an object that became completely occluded, because no image information would exist to support completion.)

Some of the work reviewed above suggests that visual memory processes might also help maintain the occluded portion of an object. Such maintenance could involve visually rehearsing information about the occluded portion of the object (e.g., Awh, Jonides, \& Reuter-Lorenz, 1998) or directing spatial attention to the occluded region. We focused on spatial short-term memory (SSTM) because of the tight linkages between this memory system and both spatial attention and spatial rehearsal (see Awh \& Jonides, 2001; Awh et al., 1998). Specifically, we asked whether either spatial attention to or SSTM for the locations later occupied by the occluded portion of an object would allow the object to be maintained behind the occluder, even in the face of strong perceptual cues that would alone support visual completion. That is, can spatial memory processes affect the maintenance of an occluded portion of an object even when image-based completion cues (e.g., T-junctions) remain fully visible? 
We used a dual-task procedure to investigate the role of SSTM in the representation of occluded objects. Observers viewed displays containing two rectangles, followed by an occluder. We measured whether the occluded portion of the rectangles was represented by using an objectbased attention task. Before the preview display, four black squares appeared, and observers were instructed to remember the locations of these squares. After ensuring that SSTM and object-based attention did not interfere with one another (Experiment 1), we manipulated the position of the to-be-remembered locations (Experiment 2). The to-be-remembered locations either did not overlap or did overlap with the locations of the occluded objects. By manipulating the location of the to-be-remembered locations, we can determine whether directing SSTM processes to the occluded regions of objects affects the maintenance of those objects.

The object-based attention task provides a measure of how strongly represented the occluded portions of the objects are; this task also allows for an indirect measure of object representation. On the critical trials, after attention was directed to one end of a rectangle, a target appeared in either the end that was aligned or unaligned with the cued end. If the occluded portion of an object was represented (i.e., maintained) behind the occluder, we should observe strong object-based effects (i.e., shorter reaction times [RTs] to targets in the aligned end than to those in the unaligned end). Weak representation of the object behind the occluder should produce weak or nonexistent objectbased effects.

Because SSTM and attention are tightly linked in some tasks (e.g., Awh et al., 1998), we first ensured that an SSTM load did not abolish object-based attentional effects. In Experiment 1, observers maintained four locations in SSTM and performed an object-based attention task on unoccluded rectangles (Experiment 1A) or on rectangles in which an "occluder" appeared behind the rectangles (Experiment 1B). In both cases, we found object-based effects, indicating that an SSTM load does not interfere with object-based attentional selection.

\section{EXPERIMENTS 1A AND 1B}

\section{Method}

Participants. There were 20 observers in each experiment. The observers were University of Iowa undergraduates who volunteered for course credit and had normal or corrected-to-normal vision.

Stimuli and Procedure. Preview displays contained two outlined rectangles that measured $1.4^{\circ} \times 8.8^{\circ}$ of visual angle from a viewing distance of $60 \mathrm{~cm}$. The rectangles were aligned either horizontally or vertically; the far edge of each rectangle was $4.4^{\circ}$ from fixation. In Experiment 1B, another occluded rectangle, measuring $1.9^{\circ} \times 10.5^{\circ}$, was added to the display to match the effects of an abruptly appearing occluder in Experiment 2 . The memory items were four black squares measuring $0.6^{\circ} \times 0.6^{\circ}$. The peripheral cue highlighted one end of a rectangle with a $0.1^{\circ}$ thick line.

For the object-based attention measure, four search stimuli were used as a target and distractors, with one shape centered at each of the four ends of the rectangles. Three small shapes $\left(0.4^{\circ}\right.$ in diameter $)$ and one large shape $\left(0.9^{\circ}\right.$ in diameter) appeared, and the target was the lone large stimulus that appeared among three small distractors.
Each target shape (circle or square) occurred equally often within each display.

The memory items that marked the to-be-remembered locations were placed randomly in a $6^{\circ} \times 6^{\circ}$ region of display centered around fixation; these locations did not overlap with the locations occupied by the rectangles in the preview display. In Experiment 1A, no occluded rectangle appeared, and in Experiment 1B, an occluded rectangle appeared behind the rectangles that contained the targets and distractors.

Figure 2A depicts the sequence of events. A fixation cross appeared for $500 \mathrm{msec}$, followed by the memory array for $1,000 \mathrm{msec}$. In Experiment 1A, no occluded rectangle appeared, so the two task-relevant rectangles appeared for an additional $500 \mathrm{msec}$. In Experiment 1B, the occluded rectangle was presented behind the task-relevant rectangles for $500 \mathrm{msec}$. Next, the cue appeared for $100 \mathrm{msec}$, followed by the target and three distractors for $50 \mathrm{msec}$. The target was the single large shape, and observers reported the target's identity as quickly and as accurately as possible via a keypress. RTs were measured from the onset of the target/distractor items. Participants responded via a keypress with their dominant hand. After a response, feedback on the response's accuracy appeared for $500 \mathrm{msec}$. The memory test array appeared after the feedback. On half of the trials, the memory test array was identical to the memory array; on the other half of the trials, one item changed its location to a new, randomly selected location. The observers made an unspeeded change/no-change response to the memory test.

The participants were given 20 practice trials, followed by 192 trials, with breaks after every 48 trials. The entire experiment lasted about $1 \mathrm{~h}$.

\section{Results and Discussion}

We analyzed only those trials with correct responses for both the target discrimination task and the memory task. RTs less than $150 \mathrm{msec}$ or greater than $2,500 \mathrm{msec}$ were excluded from the analyses ( $<1 \%$ of trials). Valid trials were not analyzed, because they were not theoretically relevant. However, across all of our experiments, the valid condition exhibited shorter RTs than did the invalid conditions, replicating the results in previous studies (Egly et al., 1994; Vecera, 1994). Valid trial RTs and accuracy data for the SSTM task appear in Table 1.

Figure 2B shows the accuracy and mean RTs for Experiments $1 \mathrm{~A}$ and $1 \mathrm{~B}$. Both experiments showed shorter RTs to targets appearing at the aligned end than at the unaligned end: Aligned end targets were discriminated faster than unaligned end targets in Experiment 1A [894 vs. $\left.920 \mathrm{msec} ; F(1,19)=4.79, p<.05, \eta^{2}=.201\right]$ and Experiment $1 \mathrm{~B}[866$ vs. $889 \mathrm{msec} ; F(1,19)=4.83, p<.05$, $\left.\eta^{2}=.203\right]$. Similar ANOVAs on the error data revealed similar results. In Experiment 1A, there was a significant error rate difference between aligned end $(9.4 \%)$ and unaligned end $(11.7 \%)$ trials $\left[F(1,19)=4.78, p<.05, \eta^{2}=\right.$ .201]. In Experiment 1B, there was no significant accuracy difference between aligned end $(9.9 \%)$ and unaligned end $(11.0 \%)$ trials $\left[F(1,19)=1.78, p>.1, \eta^{2}=.086\right]$, although the accuracy data paralleled the RT results.

Observers responded more quickly for aligned end targets than for unaligned end targets, indicating an intact object-based effect under a spatial memory load. The present results demonstrate that SSTM does not affect object-based attention in this cued discrimination task and that these two tasks can be used simultaneously to inves- 
A

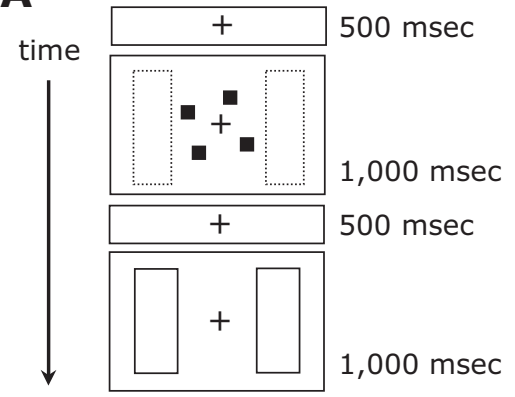

Experiment $1 \mathrm{~A}$ Experiment $1 \mathrm{~B}$
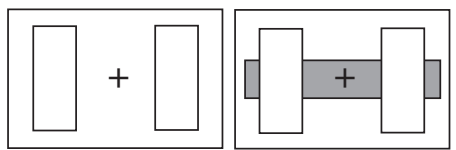

$500 \mathrm{msec}$
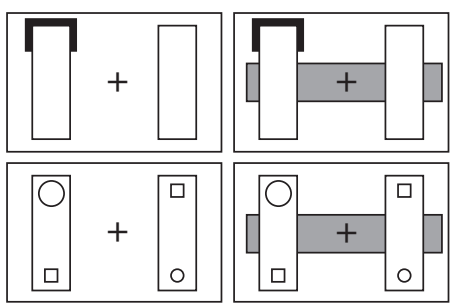

$100 \mathrm{msec}$
B

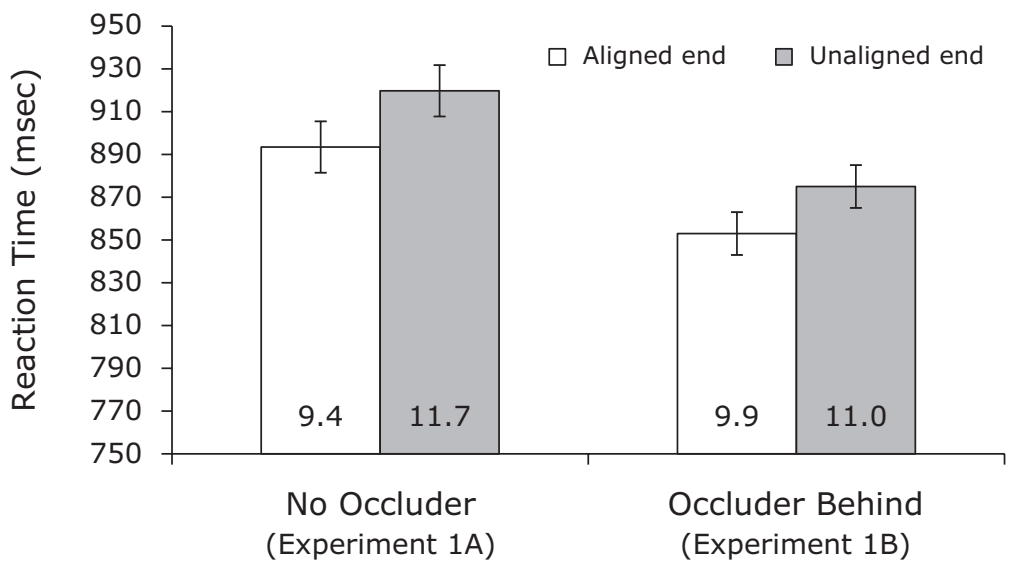

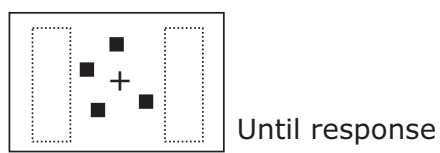

Figure 2. (A) Order of the events in Experiments 1A and 1B. Dotted lines indicate the locations occupied by the rectangles. The spatial short-term memory test display depicts a change trial on which the upper left-hand location changed. (B) Results from the invalidly cued trials in Experiments $1 \mathrm{~A}$ and $1 \mathrm{~B}$. Error bars are within-subjects $95 \%$ confidence intervals on the aligned end versus unaligned end comparisons. Numbers within the bars are the error rates for each condition.

tigate the role of SSTM rehearsal in the representation of occluded objects.

We now ask whether remembering locations that are later occupied by the occluded portions of an object allows for stronger maintenance of the occluded object than when other locations are remembered. In Experiment 2, observers remembered four locations in SSTM, but these locations fell away either from the regions occupied by occluded objects (no-overlap condition) or within the regions occupied by these objects (overlap condition). Figure $3 \mathrm{~A}$ depicts the relative locations of the memory items and the occluded objects. Importantly, in both the no-overlap and overlap conditions, there are ample image cues (T-junctions and colinearity) to support completion. If these visible cues alone dictate the representation of the occluded portion of the objects, we should find no differences between the no-overlap and overlap conditions.

In contrast, on the basis of previous studies investigating SSTM and visual attention (e.g., Awh et al., 1998), if SSTM rehearsal influences the maintenance of the occluded portions of objects, we should observe larger object-based effects when the to-be-remembered locations fall within the regions occupied by the occluded objects than when they fall away from these locations. In contrast, if only image-based cues (e.g., T-junctions) support object maintenance, we should find no differences between the overlap and no-overlap conditions.

\section{EXPERIMENT 2}

\section{Method}

Participants. The observers were 40 University of Iowa undergraduates who volunteered for course credit and had normal or corrected-to-normal vision. Half participated in the no-overlap con-

Table 1

Mean Reaction Times (RTs, in Milliseconds; With Standard Errors) and Percentages Correct for Valid Conditions and Percentages of Errors (PEs) for the Spatial Short-Term Memory (SSTM) Task in Experiments 1 and 2

\begin{tabular}{|c|c|c|c|c|c|c|}
\hline & \multicolumn{2}{|c|}{ RT } & \multicolumn{2}{|c|}{ PE } & \multicolumn{2}{|c|}{$\begin{array}{c}\text { SSTM } \\
\text { Accuracy }\end{array}$} \\
\hline & $M$ & $S E$ & $M$ & $S E$ & $M$ & $S E$ \\
\hline Experiment 1A & 838 & 40.3 & 7.9 & 1.6 & 82.3 & 1.9 \\
\hline Experiment 1B & 786 & 42.1 & 9.8 & 2.5 & 82.6 & 1.6 \\
\hline \multicolumn{7}{|l|}{ Experiment 2} \\
\hline No-overlap condition & 851 & 39.7 & 9.8 & 1.4 & 78.3 & 2.0 \\
\hline Overlap condition & 808.7 & 49.3 & 8.5 & 1.5 & 78.8 & 2.0 \\
\hline
\end{tabular}


A

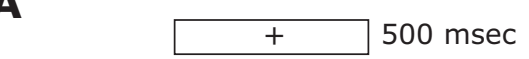

No-Overlap Condition Overlap Condition

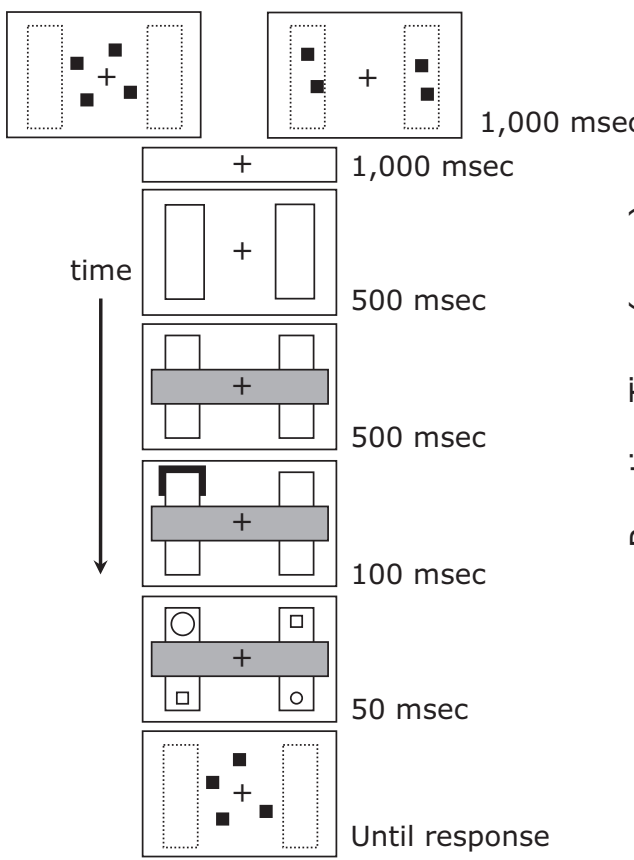

B

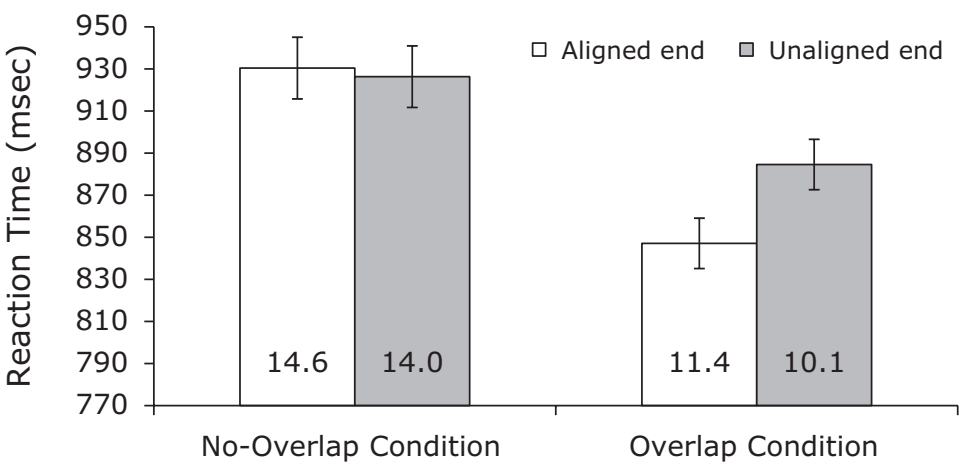

Figure 3. (A) Order of the events in Experiment 2. Dotted lines indicate the locations occupied by following rectangles. The spatial short-term memory test display depicts a change trial. (B) Results from the invalidly cued trials in Experiment 2. Error bars are within-subjects $\mathbf{9 5 \%}$ confidence intervals on aligned end versus unaligned end comparisons. Numbers within the bars are the error rates for each condition.

dition, and half in the overlap condition. We used a between-subjects manipulation to ensure that memory for one set of locations (e.g., those that overlapped the rectangles) did not affect memory for a different set of locations (e.g., those that did not overlap with the rectangles).

Stimuli and Procedure. The stimuli and procedure were similar to those in Experiment 1, with the following exceptions: First, preview displays contained three rectangles: two outlined rectangles that would be occluded (hereafter, rectangles) and an occluding rectangle (hereafter, the occluder). Second, the occluder appeared to cover the two rectangles that were visible in the preview display. Third, in the overlap condition, the memory items were located randomly at the locations occupied by the following two rectangles, extending $1.4^{\circ} \times 5.7^{\circ}$ centered at each rectangle.

\section{Results and Discussion}

We analyzed the data as in Experiment 1. RTs less than $150 \mathrm{msec}$ or greater than $2,500 \mathrm{msec}$ were excluded from the analyses $(<1 \%$ of trials). Valid trials were not analyzed, because they were not theoretically relevant. Valid trial RTs and accuracy data for the SSTM task appear in Table 1.

RTs from the invalid trials are shown in Figure 3B. These results were analyzed with a mixed-model ANOVA, with trial type (aligned end vs. unaligned end trials) as a within-subjects factor and memory location (no-overlap vs. overlap conditions) as a between-subjects factor. The analyses revealed a marginally significant main effect for trial type $\left[F(1,38)=3.3, p=.08, \eta^{2}=.079\right]$, suggesting slightly shorter RTs for aligned end trials $(889 \mathrm{msec})$ than for unaligned end trials $(906 \mathrm{msec})$. The main effect for memory location was not significant $[F(1,38)=1.1, p>$ $\left..05, \eta^{2}=.028\right]$. Importantly, the interaction between trial type and memory location was significant $[F(1,38)=5.0$, $\left.p<.05, \eta^{2}=.117\right]$.

One-factor ANOVAs investigated the object-based effects in each memory location condition. In the no-overlap condition, invalid targets in the aligned end were discriminated no faster than those appearing in the unaligned end [930 vs. $\left.926 \mathrm{msec} ; F(1,19)=.084, p>.1, \eta^{2}=.004\right]$. However, in the overlap condition, targets at the aligned end were discriminated significantly faster than those appearing at the unaligned end [ 847 vs. $885 \mathrm{msec} ; F(1,19)=$ $\left.9.78, p<.01, \eta^{2}=.340\right]$.

There were no significant effects in the error data, and there was no difference in SSTM accuracy between the no-overlap and overlap conditions. Interestingly, there was a substantial difference between the valid trials in the nooverlap and overlap conditions (see Table 1), suggesting that the location of the to-be-remembered locations might have affected the breadth of attention, which, in turn, affected the representation (viz. maintenance) of the occluded portions of objects.

Experiment 2 clearly demonstrates that SSTM influences the maintenance of the occluded portions of objects. When locations held in memory do not overlap with the occluded objects, the occluded regions are maintained 
weakly, if at all, as evidenced by the lack of an objectbased effect. Importantly, this maintenance failure occurs despite the presence of salient image-based T-junctions and colinearity cues that could have allowed the object to be maintained behind the occluder. Also, the failure to maintain the occluded objects is not due to general SSTM interference, because when memory rehearsal is directed at locations that are later occupied by the occluded portions of objects (i.e., the overlap condition), we find a reliable object-based effect. Objects are maintained behind an occluder only when SSTM processes are directed to locations that overlap with the occluded portion of an object.

\section{GENERAL DISCUSSION}

The present results, along with our previous findings (Lee \& Vecera, 2005), suggest a role for higher level visual processes in representing occluded objects. Representing and maintaining the occluded regions of objects may involve directing either SSTM rehearsal or spatial attention to locations behind the occluder. The present results indicate stronger maintenance for occluded objects when observers remembered locations that would be occupied later by the occluded portion of an object. The maintenance of occluded objects was absent when observers remembered locations that were not occupied by the occluded portion of an object. We hypothesize that in everyday viewing, objects are attended and tracked; when these objects become occluded, spatial attention, SSTM processes, and imagebased information all operate to preserve the visual representation of the occluded objects.

Previously, we suggested that VSTM for objects might directly influence completion processes themselves (Lee \& Vecera, 2005). The present results could be explained with such an interactive account in which SSTM could provide top-down feedback to the perceptual representations of objects. When to-be-remembered locations overlap with occluded objects, the additional inputs from SSTM allow an occluded object to be maintained. We should point out that noninteractive accounts are also possible. For example, SSTM and completion processes provide independent inputs to a later representation — perhaps akin to a "master map" that guides attention (e.g., Wolfe, 1994). Under such an account, completion occurs on the basis of image-based features, but the results of completion processes may be weighted less than the results of SSTM processes in ultimately guiding behavior (for relevant models, see Massaro \& Friedman, 1990; Oden \& Massaro, 1978). Alternatively, SSTM might modify the outputs of completion processes but not alter those processes themselves. Additional work will be required to distinguish the interactive and noninteractive accounts, although distinguishing such accounts can be notoriously difficult (see McClelland, 1991). But we should note that SSTM plays an active role in guiding behavior. If image cues alone affected object-based attentional search, the location of the to-be-remembered locations should not have affected the results of Experiment 2. Instead, Experiment 2 demonstrates that SSTM has a role in at least gating the outputs of completion processes, if not affecting those processes directly.

We should acknowledge that our experiments focused only on SSTM and that we did not directly examine the link between spatial attention and completion processes. Although SSTM and spatial attention are interrelated (e.g., Awh \& Jonides, 2001; Awh et al., 1998), we chose to focus on SSTM because of its relationship to other experiments that have examined memory-based inputs to completion (Lee \& Vecera, 2005). But our results could have been produced by participants' initially attending the to-be-remembered locations or maintaining those locations. However, we have initial evidence that suggests that transient spatial attention to to-be-occluded locations may be insufficient to maintain the occluded regions of objects. In our previous work, we asked participants to attend four locations (as in Experiment 2 above) and to report the highest digit; this attentional report was followed by two objects and an occluder, as in the present experiments. We found no object-based component, suggesting that the occluded objects were not maintained behind the occluder, although further work is needed to verify these preliminary findings, because they are based on a small number of observers.

The view that spatial attention and SSTM help maintain occluded objects appears to stand in contrast to several studies suggesting that visual completion occurs preattentively on the basis of image information (Davis \& Driver, 1998; He \& Nakayama, 1992; Moore et al., 2003; Rensink \& Enns, 1998) and, presumably, without the need for attention (or SSTM). Most tasks used to assess visual completion and other preattentive processes, however, rely on tasks that require attention to stimuli in a display (e.g., visual search), as when attention is directed to an occluded object to determine whether it is a target or nontarget. It is possible that attention affects completion (or its outputs) in such tasks because the stimuli are attended during search for the target, an interpretation broadly consistent with our present results. Claims regarding the relationship between spatial attention or SSTM and completion should come from tasks that make indirect measurements of completion, as in the present study and others (e.g., Moore et al., 2003; see also Kimchi \& Peterson, 2008).

A more challenging finding for the view that spatial attention and SSTM are involved in representing occluded objects comes from an inattentional blindness task. Moore et al. (2003) used inattentional blindness as an operational definition of unattended. Observers were instructed to report as quickly as possible whether the line at the center of the display was dashed or solid. The background contained unattended and task-irrelevant Pacman-like figures that could induce the perception of modally completed bars oriented either horizontally or vertically. The completed bars could cover the gaps in the dashed target line, so that the line looked a single, solid line, instead of a dashed line. The observers were slow to respond to the dashed line when it appeared to be a solid line due to the placement of the modally completed bars. This completion occurred despite observers' inability to explicitly report the identity 
of the modally completed shapes. These findings suggest that completion processes operate without attention.

Although Moore et al.'s (2003) results seem to conflict with our results, there are many differences between the two sets of results that need to be examined. For example, our present experiments examined the maintenance of an occluded object that was previously unoccluded, whereas Moore et al. investigated completion. Completion may be driven more by local bottom-up cues (e.g., T-junctions) than by influences from higher level visual processes, allowing completion to occur at unattended locations. Maintenance and rehearsal of an object that was visible initially and later occluded may require spatial attention and SSTM, although we acknowledge that there were sufficient image cues to permit completion in our displays (i.e., there were suprathreshold T-junctions present at the intersection of the rectangles and occluder). Moreover, our task relies on object-based attention as an indirect measure of the "intactness" of the occluded object. This task likely requires the occluded object to drive shifts of attention, which might require more information about the occluded object than the response competition elicited in the inattentional blindness task used by Moore et al. Finally, another relevant issue is the amount of attention consumed by the primary task in inattentional blindness paradigms. If attention is conceptualized as a graded process, a task could, in principle, be demanding enough to prevent an explicit report of unattended items, although those unattended items might receive some degree of attentional processing (see Farah, O'Reilly, \& Vecera, 1993, for a related view).

The present results have implications for our understanding of both visual completion processes and SSTM. Image-based information alone does not allow an occluded object to be maintained. SSTM may be a working memory system that operates to assist various perceptual processes, such as completion. The specific relationship between SSTM and perception remains to be worked out, however.

\section{AUTHOR NOTE}

This research was supported in part by grants from the National Science Foundation (BCS 03-39171) and the National Institutes of Health (R01AG026027). Thanks to Ed Awh, Jay Pratt, and several reviewers for their comments. Correspondence should be addressed to S. P. Vecera, Department of Psychology, University of Iowa, E11 Seashore Hall, Iowa City, IA 52242-1407 (e-mail: shaun-vecera@uiowa.edu).

\section{REFERENCES}

AwH, E., \& Jonides, J. (2001). Overlapping mechanisms of attention and working memory. Trends in Cognitive Sciences, 5, 119-126.

Awh, E., Jonides, J., \& Reuter-Lorenz, P. A. (1998). Rehearsal in spatial working memory. Journal of Experimental Psychology: Human Perception \& Performance, 24, 780-790.

Davis, G., \& Driver, J. (1998). Kanizsa subjective figures can act as occluding surfaces at parallel stages of visual search. Journal of Experimental Psychology: Human Perception \& Performance, 24, 169-184.

Egly, R., Driver, J., \& Rafal, R. D. (1994). Shifting visual attention between objects and location: Evidence from normal and parietal lesion subjects. Journal of Experimental Psychology: General, 123, 161-177.

Farah, M. J., O'Reilly, R. C., \& Vecera, S. P. (1993). Dissociated overt and covert recognition as an emergent property of a lesioned neural network. Psychological Review, 100, 571-588.

Fusita, K. (2001). Perceptual completion in rhesus monkeys (Macaca mulatta) and pigeons (Columbia livia). Perception \& Psychophysics, 63, $115-125$.

He, Z. J., \& Nakayama, K. (1992). Surfaces versus features in visual search. Nature, 359, 231-233.

Joseph, J. S., \& NAKAYAMA, K. (1999). Visual completion depends on the object seen before partial occlusion. Vision Research, 39, 283292.

KANIZSA, G. (1975). The role of regularity in perceptual organization. In G. Flores d'Arcais (Ed.), Studies in perception (pp. 48-66). Florence: Martello.

Kanizsa, G., \& Gerbino, W. (1982). Visual completion: Seeing or thinking? In J. Beck (Ed.), Organization and representation in perception (pp. 167-190). Hillsdale, NJ: Erlbaum.

Kanizsa, G., Renzi, P., Conte, S., Compostela, C., \& Guerani, L. (1993). Amodal completion in mouse vision. Perception, 22, 713721.

Kellman, P. J., \& Shipley, T. F. (1991). A theory of visual interpolation in object perception. Cognitive Psychology, 23, 141-221.

Kimchi, R., \& Peterson, M. A. (2008). Figure-ground segmentation can occur without attention. Psychological Science, 19, 660-668.

LeE, H., \& Vecera, S. P. (2005). Visual cognition influences early vision: The role of visual short-term memory in visual completion. Psychological Science, 16, 763-768.

Massaro, D. W., \& Friedman, D. (1990). Models of integration given multiple sources of information. Psychological Review, 97, 225-252.

MCClelland, J. L. (1991). Stochastic interactive processes and the effect of context on perception. Cognitive Psychology, 23, 1-44.

Michotte, A., Thinès, G., \& Crabbé, G. (1991). Amodal completion of perceptual structures. In G. Thinès, A. Costall, \& G. Butterworth (Eds.), Michotte's experimental phenomenology of perception (pp. 140-167). Hillsdale, NJ: Erlbaum. (Originally published in French in 1964)

Moore, C. M., Grosjean, M., \& Lleras, A. (2003). Using inattentional blindness as an operational definition of unattended: The case of surface completion. Visual Cognition, 10, 299-318.

Nagasaka, Y., \& Wasserman, E. A. (2008). Amodal completion of moving objects by pigeons. Perception, 37, 557-570.

ODEN, G. C., \& MASSARO, D. W. (1978). Integration of featural information in speech perception. Psychological Review, 85, 172-191.

Pratt, J., \& Sekuler, A. (2001). The effects of occlusion and past experience on the allocation of object-based attention. Psychonomic Bulletin \& Review, 8, 721-727.

RENSINK, R. A., \& ENNS, J. T. (1998). Early completion of occluded objects. Vision Research, 38, 2489-2505.

VECERA, S. P. (1994). Grouped locations and object-based attention: Comment on Egly, Driver, and Rafal (1994). Journal of Experimental Psychology: General, 123, 316-320.

Wolfe, J. M. (1994). Guided Search 2.0: A revised model of visual search. Psychonomic Bulletin \& Review, 1, 202-238.

Wouterlood, D., \& Boselie, F. (1992). A good-continuation model of some occlusion phenomena. Psychological Research, 54, 267-277.

(Manuscript received September 30, 2009; revision accepted for publication July 4, 2010.) 